\title{
Isolated pancreatic metastasis from malignant melanoma: a case report and literature review
}

\author{
Yoshifumi Nakamura ${ }^{1} \cdot$ Reiko Yamada $^{2}$ (D) Maki Kaneko ${ }^{1} \cdot$ Hiroaki Naota $^{1} \cdot$ Yu Fujimura $^{4} \cdot$ Masami Tabata $^{4} \cdot$ \\ Kazuhiko Kobayashi ${ }^{1} \cdot$ Kyosuke Tanaka $^{3}$
}

Received: 7 March 2019 / Accepted: 21 May 2019 / Published online: 27 May 2019

(c) The Author(s) 2019

\begin{abstract}
Isolated pancreatic metastasis from malignant melanoma is rare. Pancreatic metastasis is difficult to diagnose in patients with unknown primary malignant melanoma. Endoscopic ultrasound-guided fine-needle aspiration plays an important role in confirming the diagnosis. A 67-year-old woman was referred to our institution because of a mass in her pancreas. Computed tomography and magnetic resonance imaging revealed a $35-\mathrm{mm}$ mass localized on the pancreatic tail, with low attenuation, surrounded by a high-attenuation rim. Endoscopic ultrasonography revealed a hypoechoic mass with central anechoic areas. Endoscopic ultrasound-guided fine-needle aspiration of the mass was performed, and the pathological diagnosis was malignant melanoma. Intense fluorodeoxyglucose uptake was observed in the pancreatic tail on positron emission tomography-computed tomography. No other malignant melanoma was found. Distal pancreatectomy was performed. Six months postoperatively, positron emission tomography-computed tomography revealed high uptake in the left nasal cavity, and biopsy revealed the mass to be a malignant melanoma, indicating that the primary site of the malignant melanoma was the left nasal cavity and that the pancreatic mass and peritoneal lesion were metastases. The patient had survived $>2$ years after the distal pancreatectomy. Pancreatic resection of isolated pancreatic metastasis can possibly prolong survival; however, metastatic melanoma usually has poor prognosis.
\end{abstract}

Keywords Malignant melanoma $\cdot$ Endoscopic ultrasound (EUS) - Endoscopic retrograde cholangiopancreatogram $($ ERCP) $\cdot$ Endoscopic ultrasound-guided fine-needle aspiration (EUS-FNA) $\cdot$ Case report

\section{Introduction}

Pancreatic metastases are rare, ranging from 2 to $5 \%$ of pancreatic malignancies $[1,2]$. The most common primary malignancies that metastasize to the pancreas are renal, lung, breast, and colon cancer, with sarcoma and melanoma observed less commonly [2-4]. Metastatic melanoma has a

Reiko Yamada

reiko-t@clin.medic.mie-u.ac.jp

1 Department of Gastroenterology, Matsusaka Chuo General Hospital, Matsusaka, Mie, Japan

2 Department of Gastroenterology and Hepatology, Mie University Hospital, 2-174 Edobashi, Tsu, Mie 514-8507, Japan

3 Department of Endoscopy, Mie University Hospital, Tsu, Mie, Japan

4 Department of Surgery, Matsusaka Chuo General Hospital, Matsusaka, Mie, Japan poor prognosis; the median survival for patients with stage IV melanoma ranges from 8 to 18 months after diagnosis [5]. Isolated pancreatic metastasis is a rare event that represents about less than $1 \%$ of metastatic melanomas [6].

Pancreatic metastases can resemble primary pancreatic malignancies, such as ductal carcinoma or neuroendocrine tumors. Thus, it can be difficult to differentiate pancreatic metastases from primary tumors based only on imaging findings. Endoscopic ultrasound-guided fine-needle aspiration (EUS-FNA) plays an important role in confirming the diagnosis [1]. There are only a few reports on surgically resected pancreatic metastasis of malignant melanoma diagnosed by EUS-FNA [3, 7, 8]. Here, we present a unique case of malignant melanoma with isolated pancreatic metastasis diagnosed by EUS-FNA and was treated with distal pancreatectomy. 


\section{Case report}

A 67-year-old woman, who had been healthy all her life, presented to the referring hospital with left upper quadrant abdominal pain. Her ultrasonogram and computed tomography $(\mathrm{CT})$ showed a mass in the pancreas, and the patient was referred to our institution for further examination.

Enhanced CT revealed that the mass was localized to the tail of the pancreas, with pancreatic ductal dilatation. The mass was a rounded, well-defined lesion with low attenuation, surrounded by a high-attenuation rim (Fig. 1a). Magnetic resonance imaging (MRI) showed that the center of the mass was hyperintense on T1-weighted image and hypointense on T2-weighted image (Fig. 1b, c). The diffusion-weighted image showed a hyperintense peripheral rim of the mass (Fig. 1d). Endoscopic retrograde cholangiopancreatogram demonstrated smooth narrowing and displacement of the pancreatic duct with upstream dilatation (Fig. 2). EUS revealed the 35-mm mass to be hypoechoic and heterogeneous with central anechoic areas (Fig. 3a, b). Contrast-enhanced EUS (CE-EUS) was conducted using an electronic radial-type

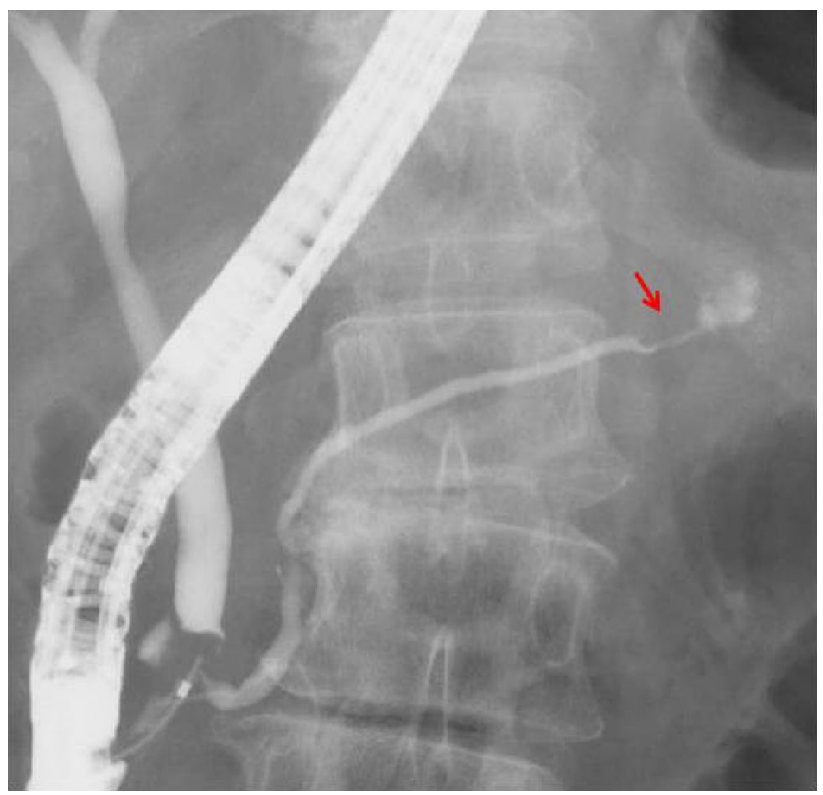

Fig. 2 Endoscopic retrograde cholangiopancreatogram revealed smooth narrowing and displacement of the pancreatic duct with upstream dilatation
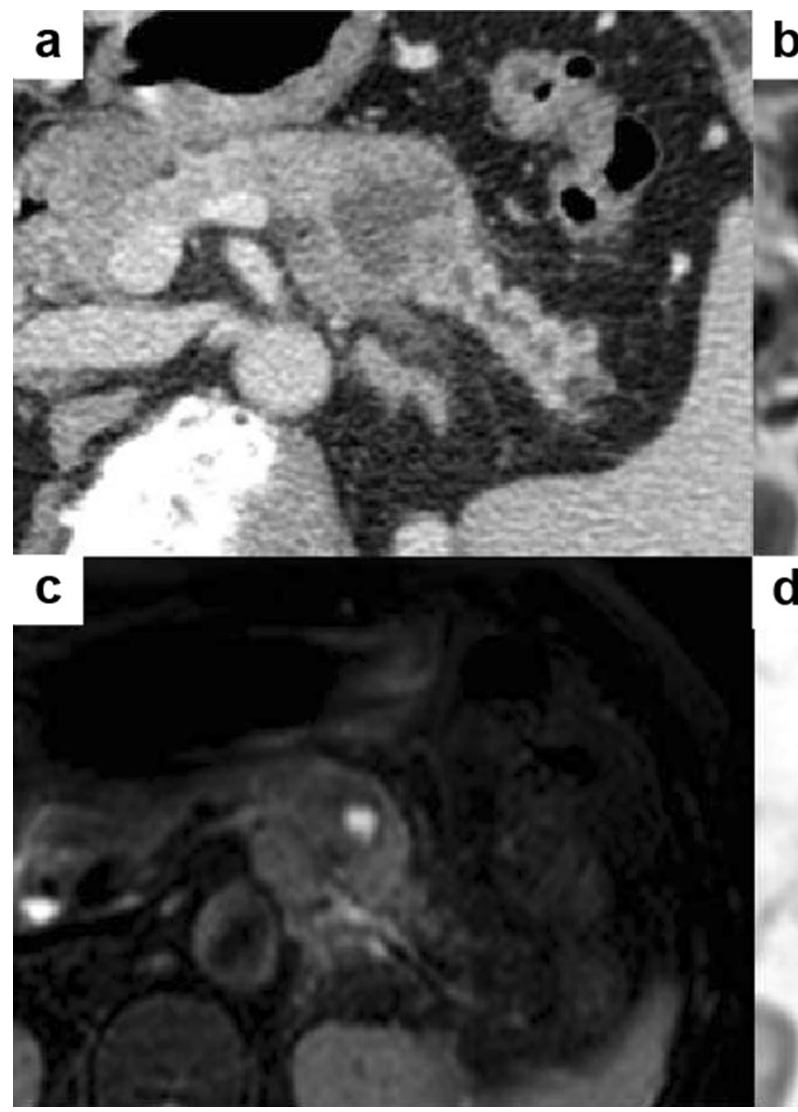

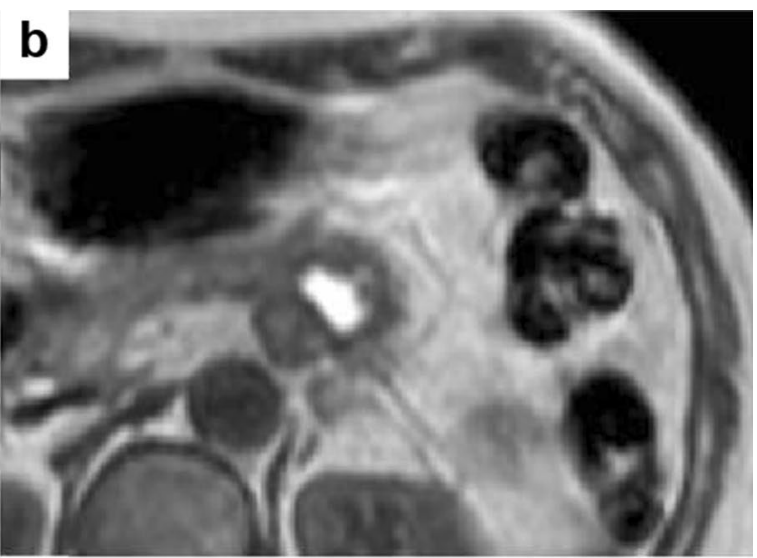

d

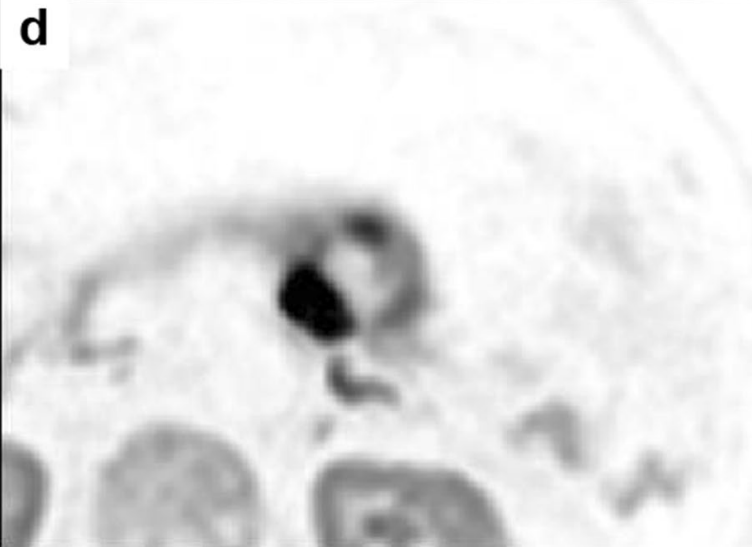

Fig. 1 Computed tomography image. a Mass in the tail of the pancreas with pancreatic ductal dilation. The central mass is hyperintense on T1-weighted image (b) and hypointense on T2-weighted image (c). d Peripheral rim of the mass is hyperintense on diffusion-weighted image 


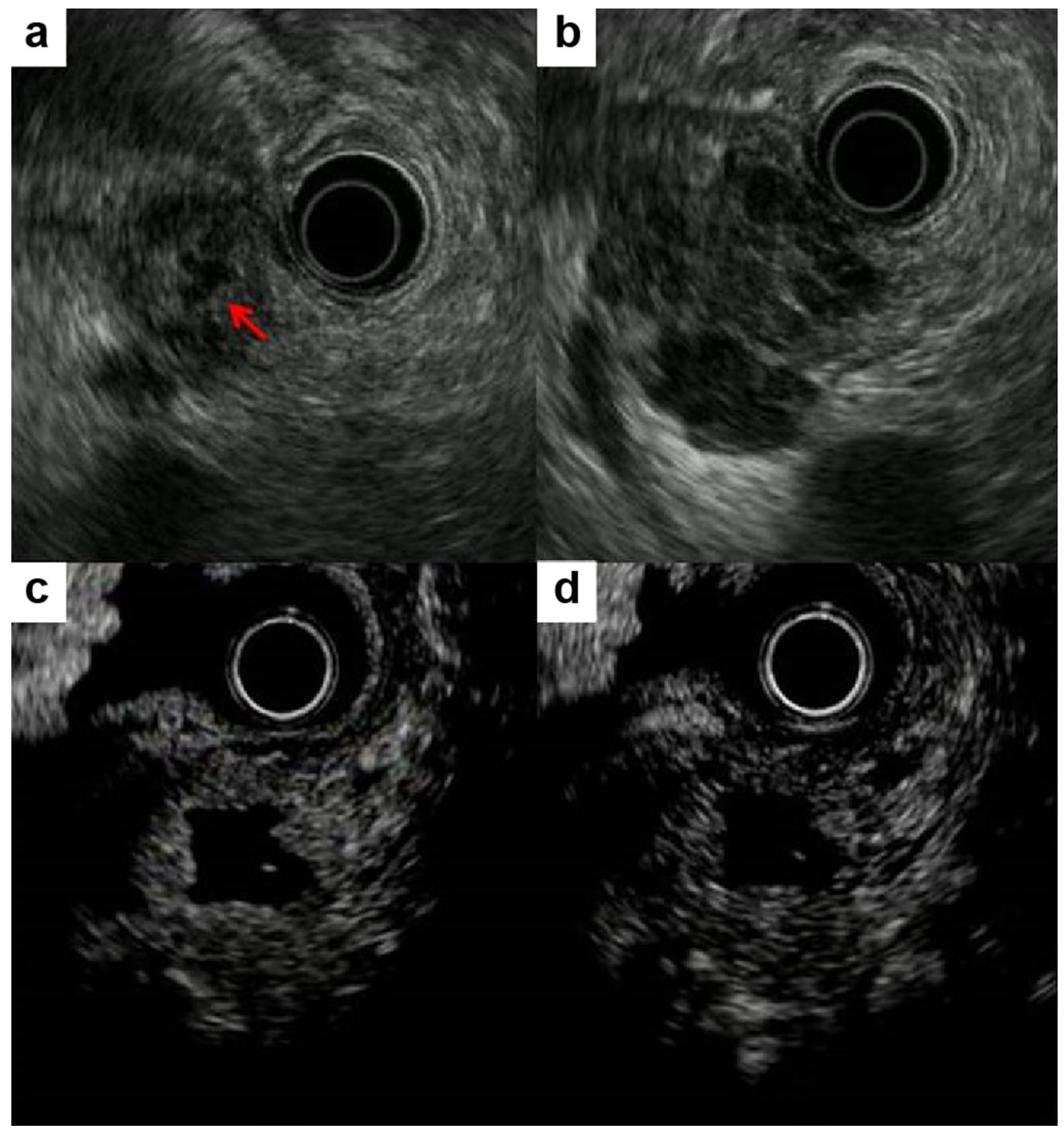

Fig. 3 Endoscopic ultrasonography revealed hypoechoic and homogenous heterogeneous mass (a) with central anechoic areas (b, arrow). Contrast-enhanced endoscopic ultrasonography shows isoenhance-

endoscope (GF-UE260; Olympus, Japan) and perflubutane as ultrasound contrast agent. CE-EUS showed isoenhancement during the 20-s phase (Fig. 3c) and hypoenhancement during the 120 -s phase (Fig. 3d) of the peripheral rim of the mass with central non-enhancement.

Cytological analysis obtained by EUS-FNA with a 22-gauge needle (Fig. 4a) revealed a large nucleus and a high nuclear/cytoplasmic ratio in the cells, with brown ment at $20 \mathrm{~s} \mathrm{(c)} \mathrm{and} \mathrm{hypoenhancement} \mathrm{at} 120 \mathrm{~s}$ (d) with central nonenhancement of the peripheral rim of the mass

pigmentation (Fig. 4b). The cells were positive for Melan A and Human Melanoma Black 45 (HMB-45) and were negative for $\mathrm{S} 100$ on cell-block immunocytochemical analysis (Fig. 4c, d). Thus, the patient was diagnosed as having malignant melanoma.

Since primary pancreatic malignant melanoma has never been reported before, we suspected metastatic malignant melanoma of the pancreas. However, intense fluorodeoxyglucose 


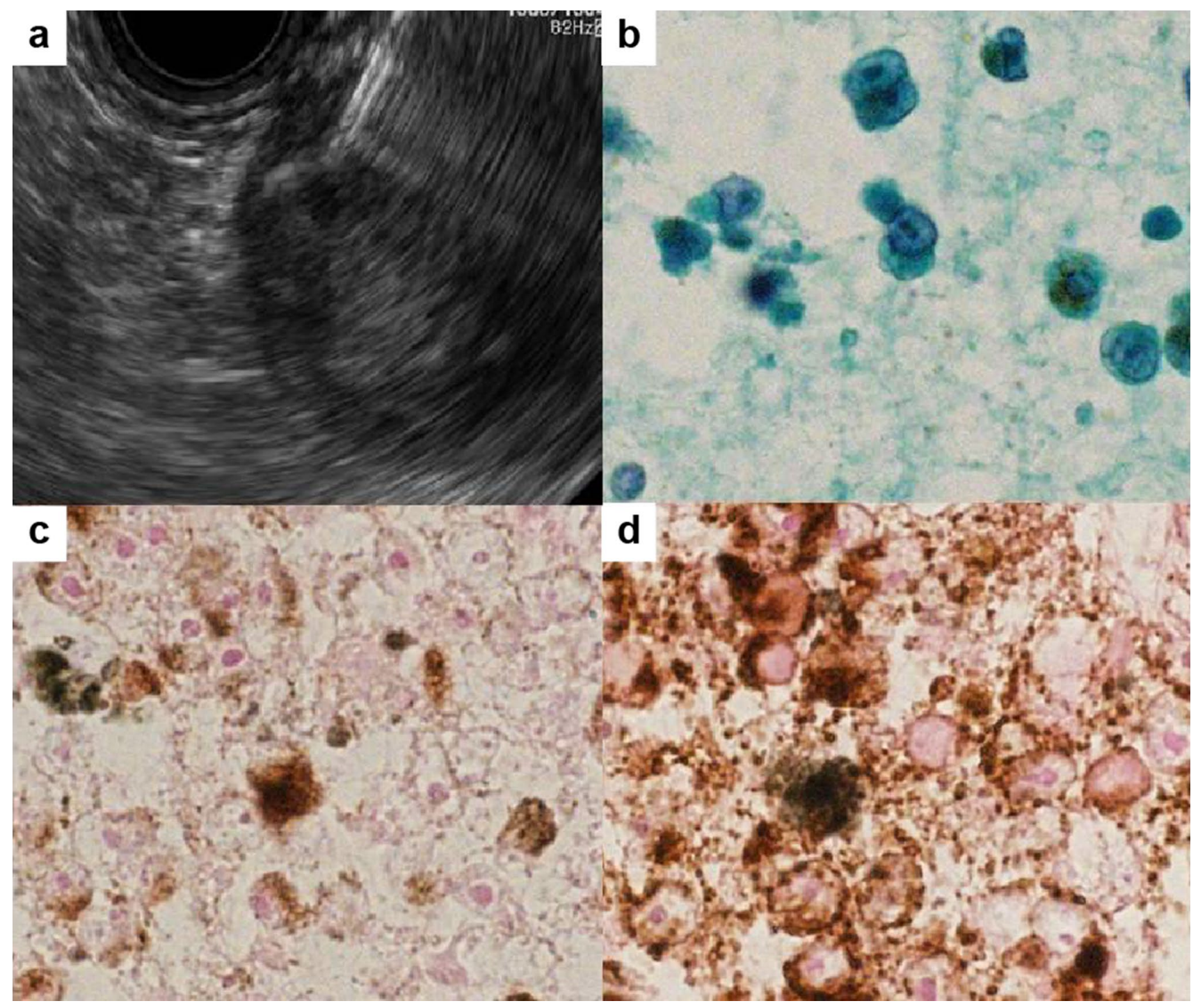

Fig. 4 a Endoscopic ultrasound-guided fine-needle aspiration of the peripheral rim of the mass. b Cytologic results revealed a large nucleus and a high nuclear/cytoplasmic ratio in the cells, with brown

uptake was observed only in the tail of the pancreas on positron emission tomography-CT (PET-CT) (Fig. 5). Esophagogastroduodenoscopy and colonoscopy did not reveal any specific findings. The primary site could not be identified by dermatological, ophthalmological, or gynecological examination.

Distal pancreatectomy was performed. Histological examination of the surgical specimen revealed malignant melanoma with central necrosis (Figs. 6, 7). The resection specimen stained for Melan A and HMB-45, but not for S100. The patient underwent interferon-alfa treatment as an adjuvant therapy. Six months postoperatively, the follow-up PET-CT pigmentation. Immunocytochemical staining with Melan A (c) and Human Melanoma Black 45 (d)

showed high uptake in the left nasal cavity, left infraclavicular lymph, and peritoneum (Fig. 8). On fiber-optic laryngoscopy, a whitish mass was detected in the left nasal cavity, which was determined to be a malignant melanoma. Although melanin was unclear in the nasal cavity biopsy specimen, cell shape and immunohistochemistry findings were the same as those in the resected surgical specimen. The primary site of the malignant melanoma was the left nasal cavity, and the pancreatic mass, left infraclavicular lymph, and peritoneal lesion were metastases. Nivolumab was started; thereafter, the treatment was 

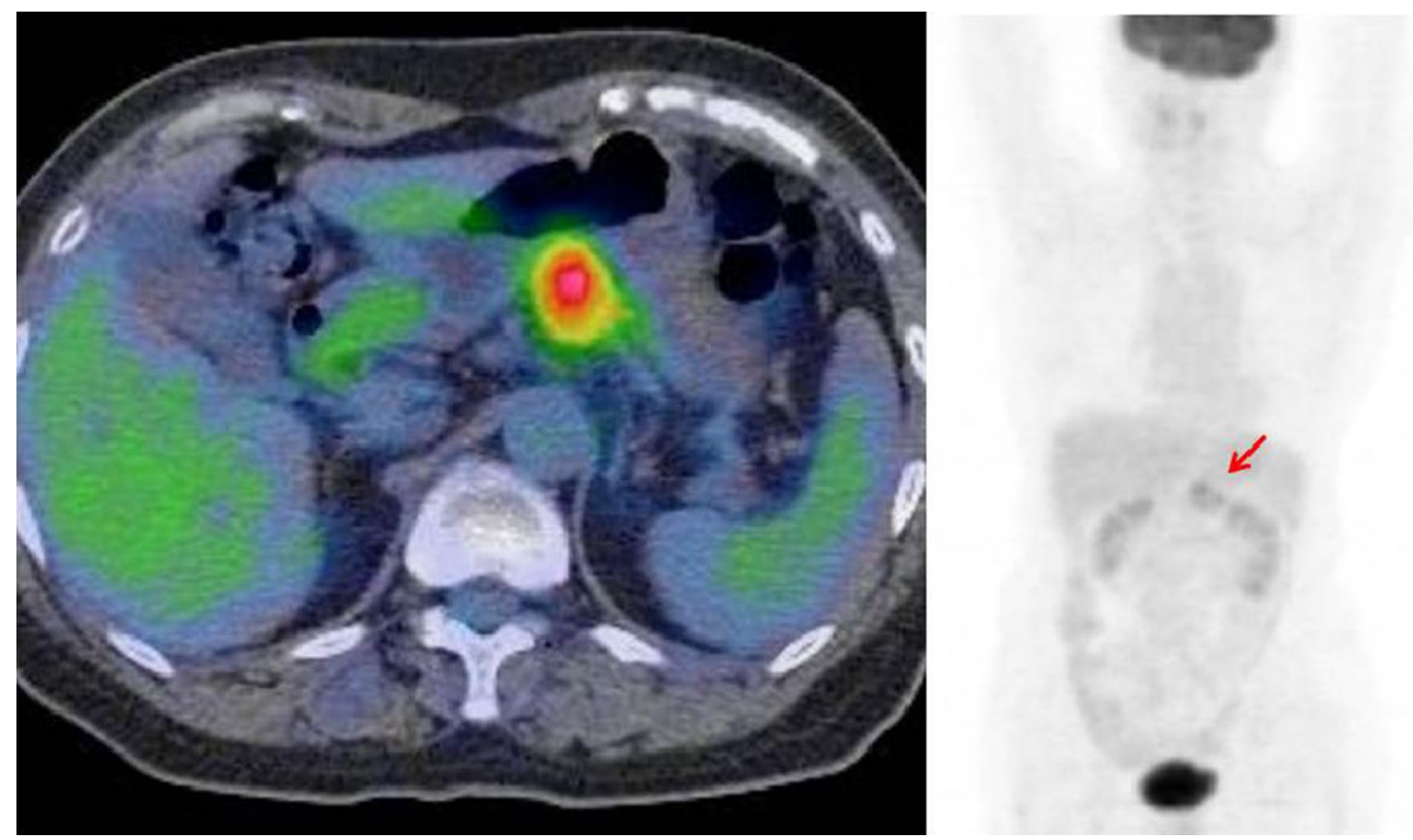

Fig. 5 Intense fluorodeoxyglucose uptake only in the body and tail of the pancreas (arrow)

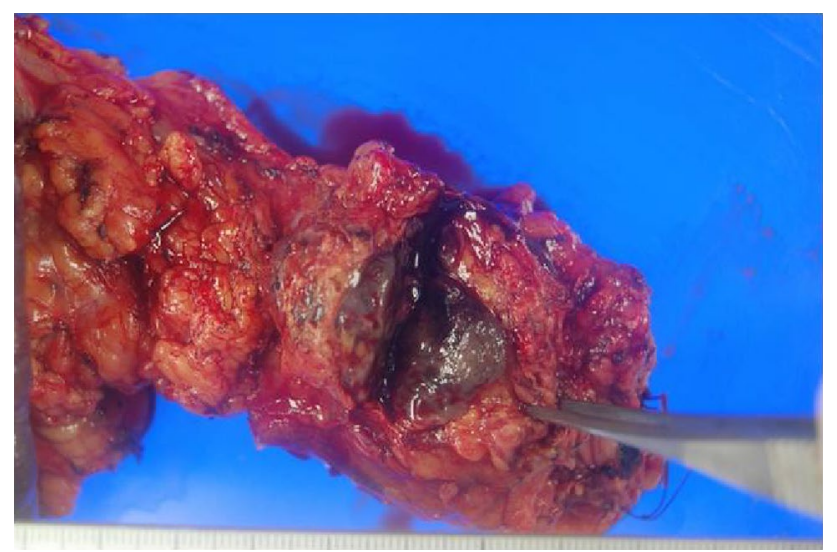

Fig. 6 Resected surgical specimen showing a black-brown mass in the tail of the pancreas

switched to pembrolizumab. The patient had survived for more than 2 years after the distal pancreatectomy.

\section{Discussion}

Malignant melanoma usually metastasizes to the gastrointestinal tract, and metastatic malignant melanoma usually affects multiple sites. Isolated organ metastasis is unusual; specifically, metastasis to the pancreas is extremely rare $(<1 \%)$ [6]. There are 76 cases of pancreatic metastasis from malignant melanoma reported in English (Table 1). The major primary site is cutaneous and ocular. Meanwhile, there are only three cases of pancreatic metastasis from nasal cavity malignant melanoma, including our case $[9,10]$. Sometimes, the primary lesion of melanoma is difficult to identify during pretreatment evaluation. In our case, the primary site was identified by PET-CT 6 months postoperatively, even though PET-CT is only effective for detecting primary tumors or cancers of unknown primary.

Despite technological advances, preoperative diagnosis of metastatic pancreatic tumor is sometimes difficult [11]. Metastatic lesions from malignant melanoma have hypervascularity on contrast-enhanced CT and MRI [12]. The blood supply to metastatic lesions is carried from the surrounding organs; therefore, the surrounding tissue of the large lesion receives more blood supply than the central area, resulting in rim enhancement, especially in lesions larger than $1.5 \mathrm{~cm}$. The same could be said in our case, as a high-attenuation rim was revealed on enhanced CT. EUS provided us with high-quality images to examine the pancreas and nearby structures. In general, pancreatic metastases on EUS have regular margins and appear as homogenous structures that are hypoechoic compared with the surrounding pancreas [13]. In our case, EUS revealed 

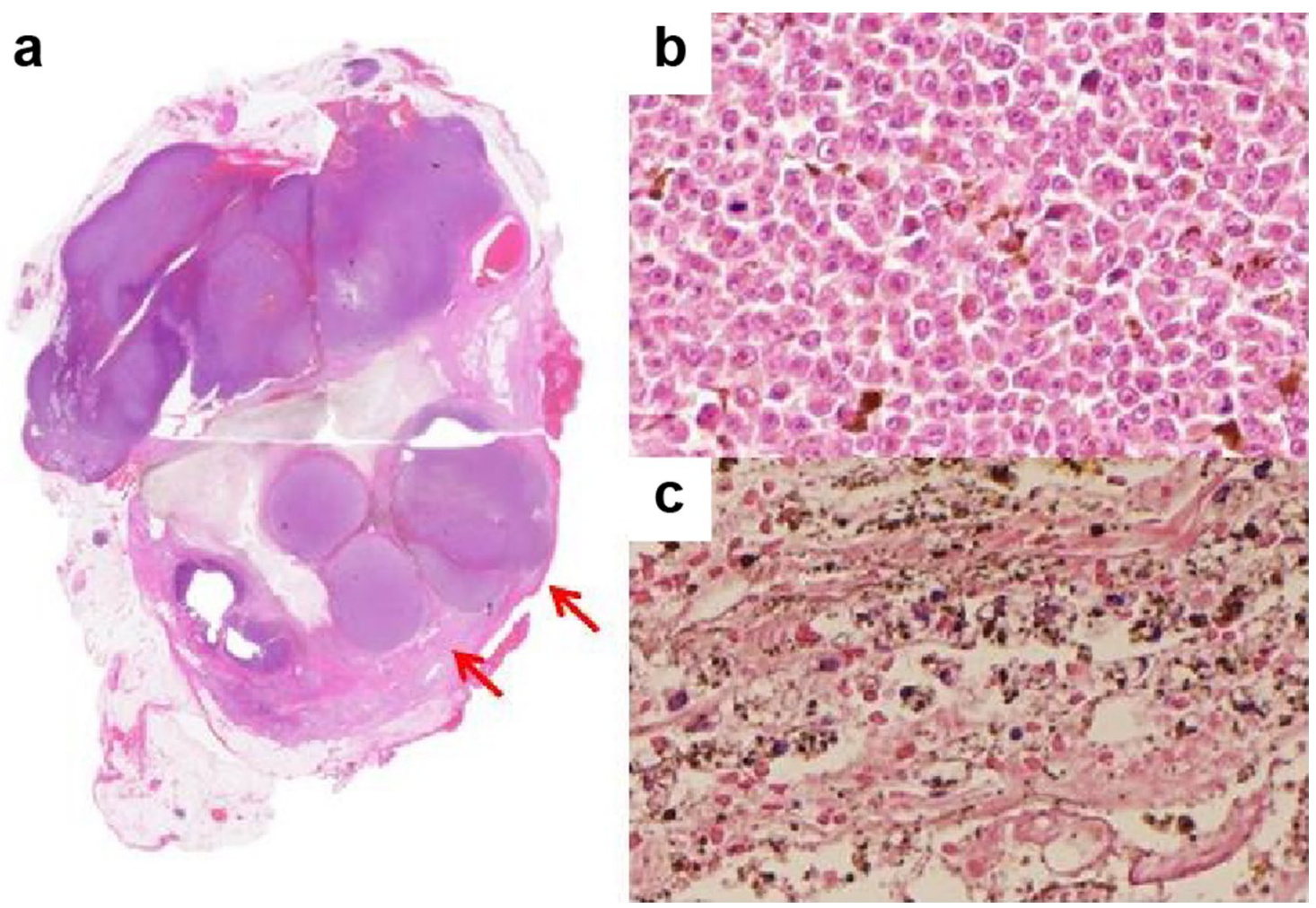

Fig. 7 a Loupe image of the resection specimen. The peripheral rim of the mass has nodular components (arrows). b Tumor cells in the peripheral rim of the mass have anisokaryosis and clear nuclei with melanin production. $\mathbf{c}$ Center of the mass was necrotic

the mass to be hypoechoic and homogenous with the central anechoic areas. Few studies have reported on CE-EUS findings of pancreatic metastatic lesion. Pancreatic metastasis of renal cell carcinoma tends to show hyperenhancement, whereas malignant melanoma may or may not show hyperenhancement [13-15]. The lack of characteristic findings makes diagnosis of metastatic malignant melanoma by CE-EUS difficult.

To confirm the diagnosis of pancreatic metastatic lesions, pathological examination is necessary. EUS-FNA plays an important role in providing cytological/histological diagnosis, and it is extremely useful in identifying pancreatic metastases. To distinguish pancreatic metastases from a primary carcinoma accurately, effective sampling and immunocytochemistry are needed $[1,3,6]$. EUS-FNA with rapid on-site evaluation provides effective sampling, because a cytopathologist can ensure that the samples are adequate for assessment [16]. Immunohistochemical analysis has been shown to be useful in identifying metastatic melanoma; the sensitivity of S100, Melan A, and HMB-45 are reported to be $97-100 \%, 75-92 \%$, and
69-93\%, respectively. The specificity of S100 and Melan A is reported to be $75-87 \%$ and $95-100 \%$, respectively [17]. In our case, Melan A and HMB-45 were positive.

The prognosis of patients with malignant melanoma metastatic to the pancreas is unknown, although metastatic melanoma usually indicates poor prognosis [5]. There are few experiences with pancreatic resection for isolated pancreatic metastases, and pancreatic resection is controversial. Some studies have shown that complete surgical resection of a localized metastatic disease can prolong survival [5, 18]. However, Wood et al. [19] reported 28 patients with isolated pancreatic metastases from malignant melanoma and found that the 5-year survival rate of pancreatic resection (performed in 8 patients) was 37.5\% (median survival, 23.8 months), as compared with $23 \%$ (median survival, 15.2 months) of the 20 patients treated with non-resection. It is critical that surgery should be performed only when a complete resection is possible. Therefore, exhaustive preoperative staging is needed to confirm both the absence of local invasion of the major vasculature and the absence of distant metastasis. PET scan has a high sensitivity and specificity 


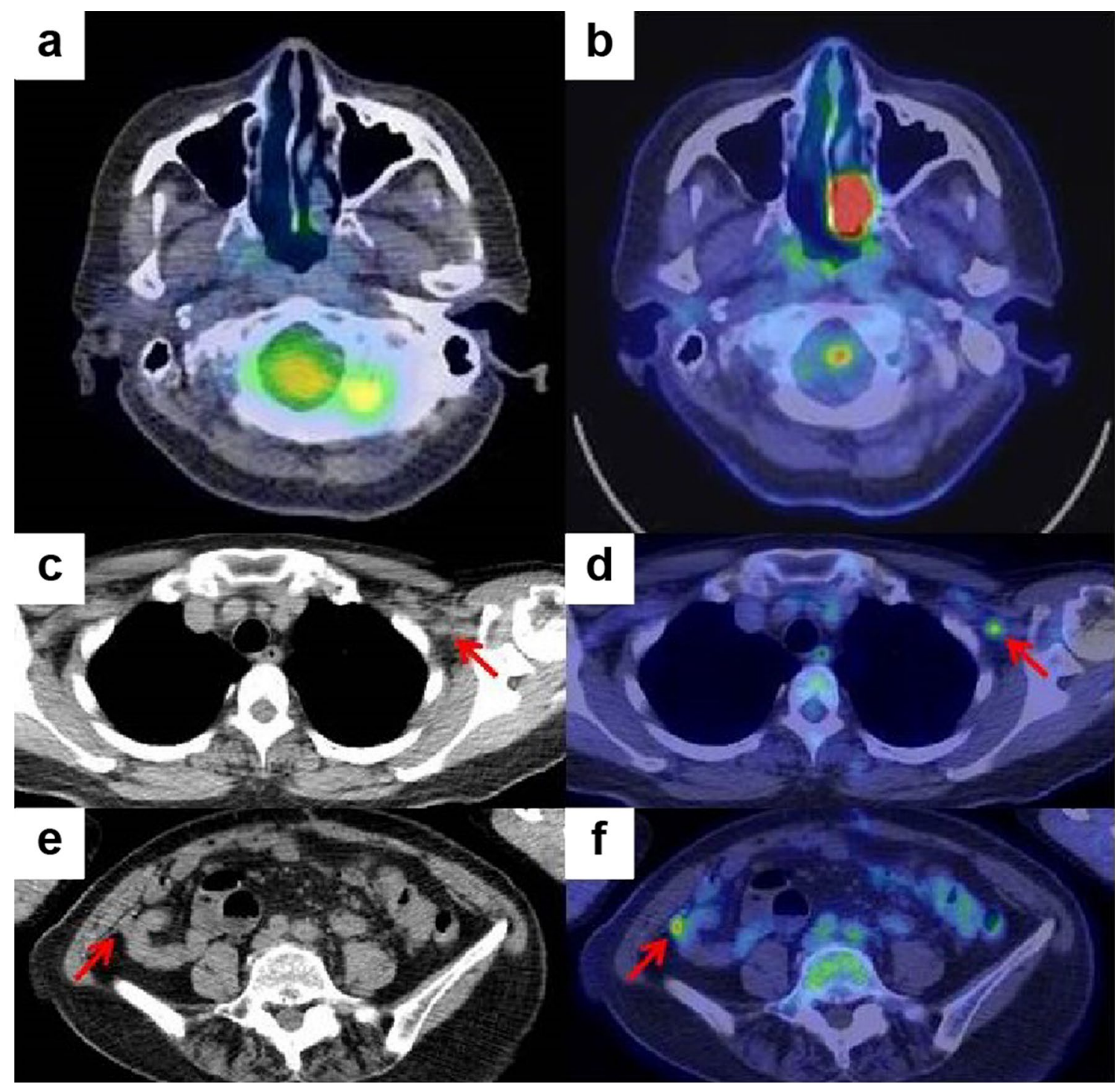

Fig. 8 Positron emission tomography-computed tomography image of the nasal cavity before (a) and after surgery (b). Plane computed tomography and positron emission tomography-computed tomog- raphy images after surgery revealed left infraclavicular lymph node metastasis $(\mathbf{c}, \mathbf{d})$ and a small peritoneal nodule $(\mathbf{e}, \mathbf{f})$

proven with EUS-FNA prior to the diagnosis of the primary lesion. Broad differential diagnoses should be considered when faced with inconclusive imaging studies of pancreatic tumors. In such cases, EUS-FNA is useful in providing a definitive diagnosis. 


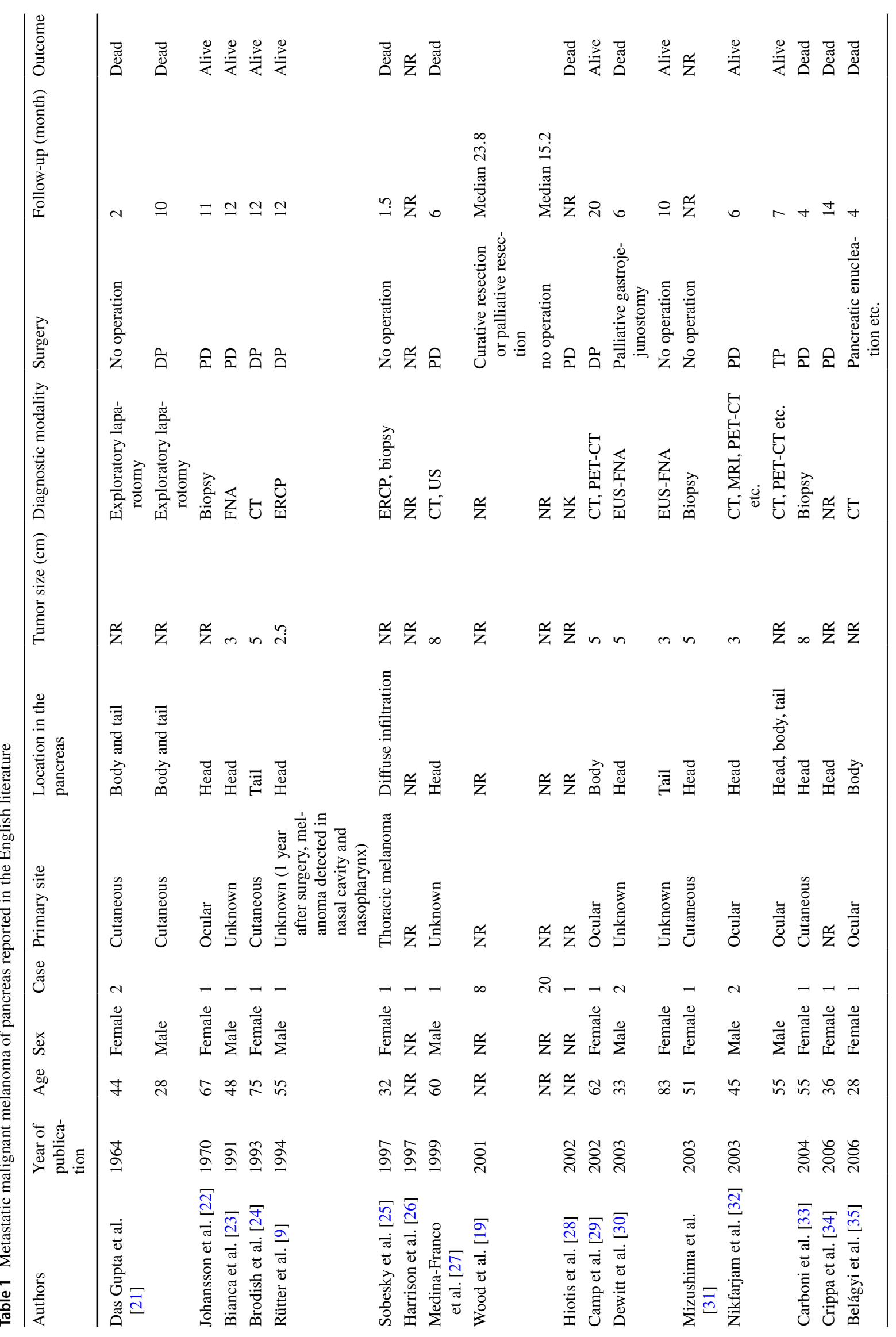




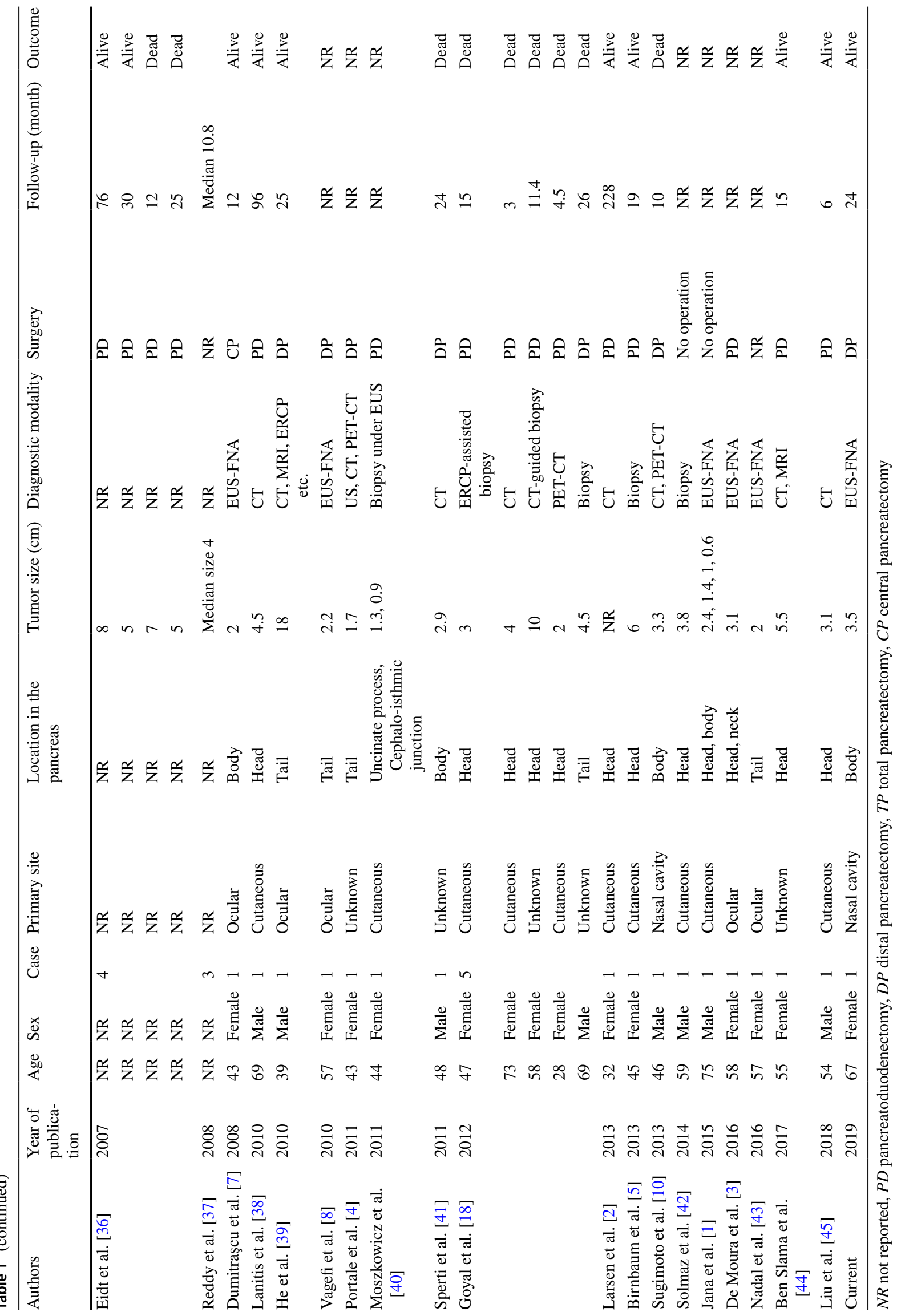


Acknowledgements We are grateful to Dr. Hiroko Sugimoto from the Department of Pathology, Matsusaka Chuo General Hospital, Matsusaka, Mie, Japan, for helpful discussions.

\section{Compliance with ethical standards}

Conflict of interest The authors declare no conflicts of interest or financial arrangement with any company.

Human rights All procedures followed were in accordance with the ethical standards of the responsible committee on human experimentation (institutional and national) and with the Helsinki Declaration of 1975, as revised in 2008(5).

Informed consent Written informed consent was obtained from the patient for publication of this case report and accompanying images.

Open Access This article is distributed under the terms of the Creative Commons Attribution 4.0 International License (http://creativeco mmons.org/licenses/by/4.0/), which permits unrestricted use, distribution, and reproduction in any medium, provided you give appropriate credit to the original author(s) and the source, provide a link to the Creative Commons license, and indicate if changes were made.

\section{References}

1. Jana T, Caraway NP, Irisawa A, et al. Multiple pancreatic metastases from malignant melanoma: conclusive diagnosis with endoscopic ultrasound-guided fine needle aspiration. Endosc Ultrasound. 2015;4:145-8.

2. Larsen AK, Krag C, Geertsen $P$, et al. Isolated malignant melanoma metastasis to the pancreas. Plast Reconstr Surg Glob Open. 2013;1:e74.

3. De Moura DT, Chacon DA, Tanigawa R, et al. Pancreatic metastases from ocular malignant melanoma: the use of endoscopic ultrasound-guided fine-needle aspiration to establish a definitive cytologic diagnosis: a case report. J Med Case Rep. 2016;10:332.

4. Portale TR, Di Benedetto V, Mosca F, et al. Isolated pancreatic metastasis from melanoma. Case report. G Chir. 2011;32:135-7.

5. Birnbaum DJ, Moutardier V, Turrini O, et al. Isolated pancreatic metastasis from malignant melanoma: is pancreatectomy worthwhile? J Surg Tech Case Rep. 2013;5:82-4.

6. Pang JC, Roh MH. Metastases to the pancreas encountered on endoscopic ultrasound-guided fine-needle aspiration. Arch Pathol Lab Med. 2015;139:1248-52.

7. Dumitraşcu T, Dima S, Popescu C, et al. An unusual indication for central pancreatectomy-late pancreatic metastasis of ocular malignant melanoma. Chirurgia. 2008;103:479-85.

8. Vagefi PA, Stangenberg L, Krings G, et al. Ocular melanoma metastatic to the pancreas after a 28-year disease-free interval. Surgery. 2010;148:151-4.

9. Rütter JE, De Graaf PW, Kooyman CD, et al. Malignant melanoma of the pancreas: primary tumour or unknown primary? Eur J Surg. 1994;160:119-20.

10. Sugimoto M, Gotohda N, Kato Y, et al. Pancreatic resection for metastatic melanoma originating from the nasal cavity: a case report and literature review. Anticancer Res. 2013;33:567-73.

11. Yagi T, Hashimoto D, Taki K, et al. Surgery for metastatic tumors of the pancreas. Surg Case Rep. 2017;3:31.
12. Tsitouridis I, Diamantopoulou A, Michaelides M, et al. Pancreatic metastases: CT and MRI findings. Diagn Interv Radiol. 2010;16:45-51.

13. Fusaroli P, D’Ercole MC, De Giorgio R, et al. Contrast harmonic endoscopic ultrasonography in the characterization of pancreatic metastases (with video). Pancreas. 2014;43:584-7.

14. Fusaroli P, Spada A, Mancino MG, Caletti G. Contrast harmonic echo-endoscopic ultrasound improves accuracy in diagnosis of solid pancreatic masses. Clin Gastroenterol Hepatol. 2010;8:629-34.

15. Yamashita Y, Kato J, Ueda K, et al. Contrast-enhanced endoscopic ultrasonography for pancreatic tumors. Biomed Res Int. 2015;2015:491782.

16. Yamao K, Sawaki A, Mizuno N, et al. Endoscopic ultrasoundguided fine-needle aspiration biopsy (EUS-FNAB): past, present, and future. J Gastroenterol. 2005;40:1013-23.

17. Ohsie SJ, Sarantopoulos GP, Cochran AJ, et al. Immunohistochemical characteristics of melanoma. J Cutan Pathol. 2008;35:433-44.

18. Goyal J, Lipson EJ, Rezaee N, et al. Surgical resection of malignant melanoma metastatic to the pancreas: case series and review of literature. J Gastrointest Cancer. 2012;43:431-6.

19. Wood TF, DiFronzo LA, Rose DM, et al. Does complete resection of melanoma metastatic to solid intra-abdominal organs improve survival? Ann Surg Oncol. 2001;8:658-62.

20. Rinne D, Baum RP, Hör G, Kaufmann R. Primary staging and follow-up of high risk melanoma patients with whole-body 18F-fluorodeoxyglucose positron emission tomography: results of a prospective study of 100 patients. Cancer. 1998;82:1664-71.

21. Gupta TD, Brasfield R. Metastatic melanoma: a clinicopathological study. Cancer. 1964;17:1323-39.

22. Johansson H, Krause U, Olding L. Pancreatic metastases from a malignant melanoma. Scand J Gastroenterol. 1970;5:573-5.

23. Bianca A, Carboni N, Di Carlo V, et al. Pancreatic malignant melanoma with occult primary lesion. A case report. Pathologica. 1991;84:531-7.

24. Brodish RJ, McFadden DW. The pancreas as the solitary site of metastasis from melanoma. Pancreas. 1993;8:276-8.

25. Sobesky R, Duclos-Vallée JC, Prat F, et al. Acute pancreatitis revealing diffuse infiltration of the pancreas by melanoma. Pancreas. 1997; 15:213-5.

26. Harrison LE, Merchant N, Cohen AM, et al. Pancreaticoduodenectomy for nonperiampullary primary tumors. Am J Surg. 1997; 174:393-5.

27. Medina-Franco H, Halpern NB, Aldrete JS. Pancreaticoduodenectomy for metastatic tumors to the periampullary region. J Gastrointest Surg. 1999;3:119-22.

28. Hiotis SP, Klimstra DS, Conlon KC, et al. Results after pancreatic resection for metastatic lesions. Ann Surg Oncol. 2002;9:675-9.

29. Camp R, Lind DS, Hemming AW. Combined liver and pancreas resection with biochemotherapy for metastatic ocular melanoma. J Hepatobiliary Pancreat Surg. 2002;9:519-21.

30. Dewitt JM, Chappo J, Sherman S. Endoscopic ultrasound-guided fine-needle aspiration of melanoma metastatic to the pancreas: report of two cases and review. Endoscopy. 2003;35:219-22.

31. Mizushima T, Tanioka H, Emori Y, et al. Metastatic pancreatic malignant melanoma: tumor thrombus formed in portal venous system 15 years after initial surgery. Pancreas. 2003;27:201-3.

32. Nikfarjam M, Evans P, Christophi C. Pancreatic resection for metastatic melanoma. HPB. 2003;5:174-9.

33. Carboni F, Graziano F, Lonardo MT, et al. Pancreaticoduodenectomy for pancreatic metastatic melanoma. J Exp Clin Cancer Res. 2004;23:539-43.

34. Crippa S, Angelini C, Mussi C, et al. Surgical treatment of metastatic tumors to the pancreas: a single center experience and review of the literature. World J Surg. 2006;30:1536-42. 
35. Belágyi $\mathrm{T}$, Zsoldos $\mathrm{P}$, Makay $\mathrm{R}$, et al. Multiorgan resection (including the pancreas) for metastasis of cutaneous malignant melanoma. J Pancreas. 2006;7:234-40.

36. Eidt S, Jergas M, Schmidt R, et al. Metastasis to the pancreasan indication for pancreatic resection? Langenbecks Arch Surg. 2007;392:539-42.

37. Reddy S, Edil BH, Cameron JL, et al. Pancreatic resection of isolated metastases from nonpancreatic primary cancers. Ann Surg Oncol. 2008;15:3199-206.

38. Lanitis S, Papaioannou N, Sgourakis G, et al. Prolonged survival after the surgical management of a solitary malignant melanoma lesion within the pancreas: a case report of curative resection. J Gastrointest Liver Dis. 2010;19:453-5.

39. He MX, Song B, Jiang H, et al. Complete resection of isolated pancreatic metastatic melanoma: a case report and review of the literature. World J Gastroenterol. 2010;16:4621-4.

40. Moszkowicz D, Peschaud F, El Hajjam M, et al. Preservation of an intra-pancreatic hepatic artery during duodenopancreatectomy for melanoma metastasis. Surg Radiol Anat. 2011;33:547-50.

41. Sperti C, Polizzi ML, Beltrame V, et al. Pancreatic resection for metastatic melanoma. Case report and review of the literature. J Gastrointest Cancer. 2011;42:302-6.
42. Solmaz A, Yigitbas H, Tokoçin M, et al. Isolated pancreatic metastasis from melanoma: a case report. J Carcinog Mutagen. 2014;5:202.

43. Nadal E, Burra P, Mescoli C, et al. Pancreatic melanoma metastasis diagnosed by endoscopic ultrasound-guided SharkCore biopsy. Endoscopy. 2016;1:E208-9.

44. Ben SS, Bacha D, Bayar R, et al. Pancreatic resection for isolated metastasis from melanoma of unknown primary. Acta Gastroenterol Belg. 2017;80:323-4.

45. Liu X, Feng F, Wang T, et al. Laparoscopic pancreaticoduodenectomy for metastatic pancreatic melanoma: a case report. Medicine. 2018;97:e12940.

Publisher's Note Springer Nature remains neutral with regard to jurisdictional claims in published maps and institutional affiliations. 